\title{
SOME NEW FIXED POINT RESULTS FOR CONVEX CONTRAC- TIONS IN B-METRIC SPACES
}

\author{
DIANA DOLIĆANIN-ĐEKIĆ ${ }^{1,2, \star}$, BANDAR BIN-MOHSIN ${ }^{3}$ \\ ${ }^{1}$ Department of Mathematics, Faculty of Technical Sciences, University of Priština, Kosovska Mitrovica, Serbia \\ ${ }^{2}$ Department of Mathematics, State university of Novi Pazar, Serbia \\ ${ }^{3}$ Department of Mathematics, College of Science, King Saud University, Riyadh 11451, Saudi Arabia
}

\begin{abstract}
The purpose of this paper is to consider various results for convex contraction mappings in the context of $B$-metric spaces. We, among other things, generalize, extend, correct and enrich the recent published results from the context of convex contractions defined on an ordinary metric spaces to the ones on the so-called $B$-metric spaces. One example shows that this generalization is genuine. Let us note that this paper represents only the beginning of our investigation of the properties of convex contractions observed in any general metric space.

In the papers that are to be published, our considerations are applied to cone metric spaces, partial metric spaces, $G$-metrics, $G_{B}$-metrics, extended $B$-metric spaces and many others.
\end{abstract}

Keywords: Convex contraction, Fixed point, $B$-metric space, $B$-Cauchy sequence

\section{INTRODUCTION AND PRELIMINARIES}

Since S. Banach proved his theorem that every contraction on a complete metric space has a unique fixed point, in his doctoral dissertation in 1922, many mathematicians have tried to generalize the famous result. Hundreds of scientific papers have considered this issue.

The previously mentioned statement, leads in two key directions: either the axioms of metric spaces are affected $\left(d_{Y}(u, v)=\right.$ 0 if and only if $u=v ; d_{Y}(u, v)=d_{Y}(v, u) ; d_{Y}(u, w) \leq$ $\left.B\left(d_{Y}(u, v)+d_{Y}(v, w)\right)\right)$, where $d_{Y}$ is a function defined on the Cartesian product $Y \times Y$, where $Y$ is a non-empty set with values in $[0,+\infty)$, or it affected the Banach contraction condition $\left(d_{Y}(f u, f v)=k d_{Y}(u, v), \forall u, v \in Y\right.$, where $\left.k \in[0,1)\right)$. More details about Banach generalization could be seen in (Aleksić et. al., 2019a), (Aleksić et. al., 2019b; Aleksić et. al., 2019c; Alghamdi et. al., 2011; Ampadu, 2017a; Ampadu, 2017b; Andras, 2003; Baakhtin, 1989; Collaco \& E Silva, 1997; Istratescu, 1981; Istratescu, 1982; Istratescu, 1983; Jeong \& Rhoades, 2005; Kirk et. al., 2003; Kirk \& Shahzad, 2014; Rhoades, 1977).

Recently, fixed point theory has been one of the most important research fields in nonlinear and functional analysis. It has wide applications in many disciplines like studying the existence of solutions for nonlinear (algebraic, differential and integral) equations, a system of linear (nonlinear) equations and convergence of many computational methods, economics, sports, medical sciences, etc.

Throughout this paper, $\mathbb{N}$ denotes the set of all positive integers. We repeat some definitions and results, which will be needed in the sequel.
Definition 1. Let $Y$ be a (non-empty) set and $B \geq 1$ a given real number. A function $d_{Y}: Y \times Y \rightarrow[0,+\infty)$ is said to be a $B$-metric on $Y$ if the following conditions are satisfied:

$\left(\mathrm{b}_{1}\right) d_{Y}(u, v)=0$ if and only if $u=v$;

$\left(\mathrm{b}_{2}\right) d_{Y}(u, v)=d_{Y}(v, u)$ for all $u, v \in Y$;

$\left(\mathrm{b}_{3}\right) d_{Y}(u, w) \leq B\left(d_{Y}(u, v)+d_{Y}(v, w)\right)$ for all $u, v, w \in Y$.

The triplet $\left(Y, d_{Y}, B\right)$ is called a $B$-metric space with coefficient $B$.

For more notions such as $B$-continuous, $B$-convergence, $B$ completeness, $B$-Cauchy sequence, all in the framework of $B$ metric spaces, the reader is referred to (Aleksić et. al., 2019a; Aleksić et. al., 2019b; Aleksić et. al., 2019c; Ampadu, 2017a; Baakhtin, 1989; Kirk \& Shahzad, 2014).

For example, the sequence $\left\{u_{n}\right\}$ in $B$-metric space $\left(Y, d_{Y}\right)$ converges to the point $u \in Y$ if for each positive number $\eta$ there is a natural number $k_{0}$ such that when $n \geq k_{0}$ we have $d_{Y}\left(u_{n}, u\right)<\eta$. This can be also written as $u_{n} \rightarrow u$ when $n \rightarrow \infty$ or even as $\lim _{n \rightarrow \infty} d_{Y}\left(u_{n}, u\right)=0$.

The sequence in $B$-metric space $\left(Y, d_{Y}\right)$ is $B$-Cauchy if the following is satisfied: For each positive number $\eta$ there is a natural number $k_{0}$ such that when $m, n \geq k_{0}$ we have $d_{Y}\left(u_{n}, u_{m}\right)<\eta$. We can also write the last expression in the form $\lim _{m, n \rightarrow \infty} d_{Y}\left(u_{n}, u_{m}\right)=0$.

We say that the $B$-metric space is $B$-complete if every $B$ Cauchy sequence in it converges.

Let us note that every $B$-convergent sequence in the $B$-metric space $\left(Y, d_{Y}\right)$ is also $B$-Cauchy. Indeed, this fact follows simply from the next inequality:

$$
\frac{1}{B} d_{Y}\left(u_{n}, u_{m}\right) \leq d_{Y}\left(u_{n}, u\right)+d_{Y}\left(u, u_{m}\right) .
$$


It is well-known that in every ordinary metric space $\left(M, d^{M}\right)$ the metric $d^{M}$ is a continuous function with two variables. This means that $d^{M}\left(a_{n}, b_{n}\right) \rightarrow d^{M}(a, b)$ when $n \rightarrow \infty$ whenever $a_{n} \rightarrow a$ and $b_{n} \rightarrow b$ in the case when $n \rightarrow \infty$.

However, it is not the case in $B$-metric spaces, and this is one of the most important properties that distinguishes $B$-metric spaces (if $B>1$ ) from metric spaces $(B=1)$.

The following, well-known, example in the literature shows this.

Example 2. Let $Y=\mathbb{N} \cup\{\infty\}$ and $d_{Y}: Y \times Y \rightarrow[0,+\infty)$ be defined in the following way

$$
d_{Y}(p, q)= \begin{cases}0, & \text { if } p=q, \\ \left|\frac{1}{p}-\frac{1}{q}\right|, & \text { if } p, q \text { are even, or } p q=\infty, \\ 5, & \text { if } p, q \text { are odd, and } p \neq q \\ 2, & \text { in other cases. }\end{cases}
$$

It is easy to verify that the triangle relation is fulfilled in the context of the $B$-metric space for $B=3$, i.e.,

$$
d_{Y}(p, q) \leq 3\left(d_{Y}(p, r)+d_{Y}(r, q)\right),
$$

for all $p, q, r \in Y$. The other two axioms are obviously satisfied.

We check that the $B$-metric $d_{Y}$ is not $B$-continuous $(B=3)$.

Indeed, if $a_{n}=2 n$, for each $n \in \mathbb{N}$, then $d_{Y}\left(a_{n}, \infty\right)=$ $d_{Y}(2 n, \infty) \rightarrow 0$, when $n \rightarrow \infty$, i.e., $a_{n} \rightarrow \infty$, but it is obvious that $d_{Y}\left(a_{n}, 1\right)=2$ is not $d_{Y}(\infty, 1)$, when $n \rightarrow \infty$.

Note that the previous example shows that there exists a $B$ metric $d_{Y}$ which is not a metric.

It is important to know how $B$-metrics can be obtained from the ordinary metric. In that case, the obtained $B$-metric is a continuous function with two variables. So we have the following:

Let $\left(M, d^{M}\right)$ be a given metric space and let $s>1$ be a given real number. Then, with $d_{M}(u, v)=\left(d^{M}(u, v)\right)^{s}$ one $B$-metric is defined on a non-empty set $M$, where $B=2^{s-1}$. Obviously, $d_{M}$ is a continuous $B$-metric on the set $M$.

In order to prove a more general result than the previous one, a well-known property of the real function $x \mapsto x^{b}, x>0, b>1$, is used and it is given in the form

$$
\left(\frac{u+v}{2}\right)^{b} \leq \frac{u^{b}+v^{b}}{2}
$$

whenever $u$ and $v$ are positive numbers and $b>1$.

Now, let's state this well-known result:

Let $\left(M, d^{M}\right)$ be a given metric space and let $b>1, \lambda \geq 0$, $\alpha>0, u, v \in M$. We define the mapping

$$
d_{M}(u, v)=\lambda d^{M}(u, v)+\alpha\left(d^{M}(u, v)\right)^{b} .
$$

We have that the function $d_{M}$ is not the ordinary metric, but $\left(M, d^{M}\right)$ is $B$ - metric space, where coefficient $B=2^{b-1}$. Indeed, since it is sufficient to check only the triangle relation (the first two axioms are proved trivial) by applying the previous property of the convex function $x \mapsto x^{b}, x>0, b>1$, we have

$$
\begin{gathered}
d_{M}(u, v)=\lambda d^{M}(u, v)+\alpha\left(d^{M}(u, v)\right)^{b} \leq \lambda\left(d^{M}(u, w)+d^{M}(w, v)\right)+ \\
+\alpha\left(\left(d^{M}(u, w)\right)^{b}+\left(d^{M}(w, v)\right)^{b}\right) \leq \lambda\left(d^{M}(u, w)+d^{M}(w, v)\right)+ \\
+2^{b-1} \alpha\left(\left(d^{M}(u, w)\right)^{b}+\left(d^{M}(w, v)\right)^{b}\right) \leq 2^{b-1}\left(d^{M}(u, w)+d^{M}(w, v)\right) .
\end{gathered}
$$

Example 3. Let $t \in(0,1)$ and let

$$
Y=l_{t}(\mathbb{R}):=\left\{u=\left\{u_{n}\right\} \subset \mathbb{R}: \sum_{n=1}^{+\infty}\left|u_{n}\right|^{t}<\infty\right\} .
$$

For $u=\left\{u_{n}\right\}, v=\left\{v_{n}\right\} \in Y$, we define

$$
d_{M}(u, v)=\left(\sum_{n=1}^{+\infty}\left|u_{n}-v_{n}\right|^{t}\right)^{\frac{1}{t}}
$$

Using the previous property it is easy to show that $\left(Y, d_{M}\right)$ is a $B$-metric space with coefficient $B=2^{\frac{1}{t}}$.

At the end of this introductory section, we note the following result that has recently been one of the most popular in the works from the context of the $B$-metric space:

- If for some sequence $\left\{u_{n}\right\}$ in the $B$-metric space $\left(Y, d_{Y}\right)$ is satisfied the inequality:

$$
d_{M}\left(u_{n+1}, u_{n}\right) \leq \mu d_{M}\left(u_{n}, u_{n-1}\right),
$$

for each $n \in \mathbb{N}$, where $\mu \in[0,1)$, then the sequence $\left\{u_{n}\right\}$ is $B$ Cauchy. Otherwise, in the general case, the following auxiliary result is most often used:

- Let $\left(Y, d_{Y}\right)$ be a given $B$-metric space with a coefficient $B \geq$ 1. Suppose that $\left\{u_{n}\right\}$ and $\left\{v_{n}\right\}$ are given sequences that respectively converge to a points $u$ and $v$. Then the following applies

$\frac{1}{B^{2}} d_{Y}(u, v) \leq \lim _{n \rightarrow \infty} \inf d_{Y}\left(u_{n}, v_{n}\right) \leq \lim _{n \rightarrow \infty} \sup d_{Y}\left(u_{n}, v_{n}\right) \leq B^{2} d_{Y}(u, v)$.

If, in the previous inequality we put that $u=v$, we obtained $\lim _{n \rightarrow \infty} d_{Y}\left(u_{n}, v_{n}\right)=0$. Moreover, in that case, for every $w$ we have $\frac{1}{B} d_{Y}(u, w) \leq \lim _{n \rightarrow \infty} \inf d_{Y}\left(u_{n}, w\right) \leq \lim _{n \rightarrow \infty} \sup d_{Y}\left(u_{n}, w\right) \leq B d_{Y}(u, w)$.

\section{MAIN RESULTS}

In this note we will consider the next contractive condition defined in the context of so-called $B$-metric spaces. For $B$ metric spaces see (Aleksić et. al., 2019a; Ampadu, 2017a; Kirk $\&$ Shahzad, 2014). Let $Y$ be a non-empty set and $d_{Y}$ be a metric. Recall from Istratescu (Istratescu, 1981) that a map $I: Y \rightarrow Y$ is called a convex contraction mapping of order 2 , if for all $u, v \in Y$, $p, q \geq 0, p+q<1$, it holds that

$$
d_{Y}\left(I^{2} u, I^{2} v\right) \leq p d_{Y}(I u, I v)+q d_{Y}(u, v) .
$$

Alternatively, one could say $I: Y \rightarrow Y$ is a convex type contraction mapping of order 2 , if for all $u, v \in Y$ and $0 \leq k \leq \frac{1}{4}$ it holds that

$$
d_{Y}\left(I^{2} u, I^{2} v\right) \leq k\left[d_{Y}(I u, I v)+d_{Y}(u, v)\right] .
$$


In the case that $\left(Y, d_{Y}\right)$ is a $B$-metric space with the coefficient $B \geq$ 1 then $p, q \geq 0, p+q<\frac{1}{B^{2}}$, that is, $p+q<\frac{1}{4 B^{2}}$ if eq. (2) holds.

Our following results improve ones from from (Ampadu, 2017a) and (Ampadu, 2017b).

Theorem 4. Let $\left(Y, d_{Y}\right)$ be a $B$-complete $B$-metric space with the coefficient $B \geq 1$ and let $I: Y \rightarrow Y$ be a $B$-continuous mapping such that for all $u, v \in Y$ eq. (1) holds, where $p, q \geq 0, p+q<\frac{1}{B^{2}}$. Then $I$ has a unique fixed point (say $z \in Y$ ) and for all $u \in Y$ the sequence $\left\{I^{n} u\right\}_{n=1}^{+\infty}$ converges to the fixed point $z$.

Proof. The uniqueness is clear simple and therefore we prove only the existence.

Let $z_{0}$ be an arbitrary but fixed point in $Y$ and consider the orbit of $z_{0}$ under $I$, that is, the set $\left(I^{n}\left(z_{0}\right)\right)_{0}^{+\infty}, I^{0}\left(z_{0}\right)=z_{0}$. Set $R=\max \left\{d_{Y}\left(z_{0}, I\left(z_{0}\right)\right), d_{Y}\left(I\left(z_{0}\right), I^{2}\left(z_{0}\right)\right)\right\}$. Thus for any $m \geq 2$

$$
\begin{array}{r}
d_{Y}\left(I^{2 m+1}\left(z_{0}\right), I^{2 m}\left(z_{0}\right)\right) \\
\leq p d_{Y}\left(I^{2 m}\left(z_{0}\right), I^{2 m-1}\left(z_{0}\right)\right) \\
+q d_{Y}\left(I^{2 m-1}\left(z_{0}\right), I^{2 m-2}\left(z_{0}\right)\right)
\end{array}
$$

and

$$
\begin{array}{r}
d_{Y}\left(I^{2 m-1}\left(z_{0}\right), I^{2 m}\left(z_{0}\right)\right) \\
\leq p d_{Y}\left(I^{2 m-2}\left(z_{0}\right), I^{2 m-1}\left(z_{0}\right)\right) \\
+q d_{Y}\left(I^{2 m-3}\left(z_{0}\right), I^{2 m-2}\left(z_{0}\right)\right) .
\end{array}
$$

The condition (3) implies the next relations:

$$
\begin{gathered}
d_{Y}\left(I^{3}\left(z_{0}\right), I^{2}\left(z_{0}\right)\right) \leq p d_{Y}\left(I^{2}\left(z_{0}\right), I^{1}\left(z_{0}\right)\right) \\
+q d_{Y}\left(I^{1}\left(z_{0}\right), z_{0}\right) \leq R(p+q) \\
+q d_{Y}\left(I^{2}\left(z_{0}\right), I\left(z_{0}\right)\right) \leq p R(p+q)+q R \leq R(p+q), \\
\left.d_{Y}\left(I^{5}\left(z_{0}\right), I^{4}\left(z_{0}\right)\right) \leq p d_{Y}\left(I^{4}\left(z_{0}\right)\right) \leq d_{Y}\left(I^{3}\left(z_{0}\right), I^{2}\left(z_{0}\right)\right)\right) \\
+q d_{Y}\left(I^{3}\left(z_{0}\right), I^{2}\left(z_{0}\right)\right) \leq p R(p+q) \\
+q R(p+q)=R(p+q)^{2},
\end{gathered}
$$

because $p+q<\frac{1}{B^{2}} \leq 1$.

An induction argument shows that

$$
d_{Y}\left(I^{2 m+1}\left(z_{0}\right), I^{2 m}\left(z_{0}\right)\right) \leq R(p+q)^{m}<\frac{R}{B^{2 m}}
$$

and the similar by eq. (4) that

$$
d_{Y}\left(I^{2 m-1}\left(z_{0}\right), I^{2 m}\left(z_{0}\right)\right) \leq R(p+q)^{m}<\frac{R}{B^{2 m}},
$$

in the case that $B>1$. If $B=1$ the proof follows by (Istratescu, 1981).

Now according (Aleksić et al., 2019a, Remark 2.1.) for $m<$ $n$ follows

$$
\begin{array}{r}
d_{Y}\left(I^{m}\left(z_{0}\right), I^{n}\left(z_{0}\right)\right) \leq B d_{Y}\left(I^{m}\left(z_{0}\right), I^{m+1}\left(z_{0}\right)\right) \\
+B^{2} d_{Y}\left(I^{m+1}\left(z_{0}\right), I^{m+2}\left(z_{0}\right)\right)+\cdots+
\end{array}
$$

$$
\begin{gathered}
+B^{n-m-1} d_{Y}\left(I^{n-2}\left(z_{0}\right), I^{n-1}\left(z_{0}\right)\right) \\
+B^{n-m-1} d_{Y}\left(I^{n-1}\left(z_{0}\right), I^{n}\left(z_{0}\right)\right) .
\end{gathered}
$$

Further from (7) we obtain that $I^{n}\left(z_{0}\right)$ is a $B$-Cauchy sequence in $B$-metric space $\left(Y, d_{Y}\right)$ with the coefficient $B>1$.

Indeed, if $m=2 k, n=2 l(m=2 k, n=2 l-1$ or $m=2 k-1$, $n=2 l$ ), then by using (5), (6) and (7) the result follows. Since $\left(Y, d_{Y}\right)$ is a $B$-complete $B$-metric space, then there exists $z \in Y$ such that $u_{n} \rightarrow z$ as $n \rightarrow \infty$, i.e., $I z=z$ because $I$ is $B$-continuous mapping. The proof of Theorem 4 is complete.

Let for example $m=4, n=10$, i.e., $k=2, l=5$. Then

$$
\begin{array}{r}
d_{Y}\left(I^{4}\left(z_{0}\right), I^{10}\left(z_{0}\right)\right) \leq B d_{Y}\left(I^{4}\left(z_{0}\right), I^{5}\left(z_{0}\right)\right) \\
+B^{2} d_{Y}\left(I^{5}\left(z_{0}\right), I^{6}\left(z_{0}\right)\right)+B^{3} d_{Y}\left(I^{6}\left(z_{0}\right), I^{7}\left(z_{0}\right)\right) \\
+B^{4} d_{Y}\left(I^{7}\left(z_{0}\right), I^{8}\left(z_{0}\right)\right)+B^{5} d_{Y}\left(I^{8}\left(z_{0}\right), I^{9}\left(z_{0}\right)\right) \\
+B^{5} d_{Y}\left(I^{9}\left(z_{0}\right), I^{10}\left(z_{0}\right)\right) \leq 2 B(p+q)^{2} \\
+2 B^{3}(p+q)^{3}+2 B^{5}(p+q)^{4}+2 B^{7}(p+q)^{5}= \\
2 B(p+q)^{2} \frac{1-\left[B^{2}(p+q)\right]^{4}}{1-B^{2}(p+q)} \leq 2 B(p+q)^{2}(1+\cdots) .
\end{array}
$$

Hence, follows the method for general case, for example $m=2 k, n=2 l$.

Corollary 5. (Istratescu, 1981, Theorem 1.2.) Let $\left(Y, d_{Y}\right)$ be a complete metric space and let $I: Y \rightarrow Y$ be a continuous mapping such that for all $u, v \in Y$ eq. (1) holds, where $p, q \geq 0, p+q<1$. Then $I$ has a unique fixed point (say $z \in Y$ ) and for all $u \in Y$ the sequence $\left\{I^{n} u\right\}_{n=1}^{+\infty}$ converges to the fixed point $z$.

Remark 6. It is worth to notice that our proof given in Theorem 4 improves the corresponding from (Ampadu, 2017, Theorem 8). This means that our Theorem 4 is a new result for convex contractions in the context of $B$-metric spaces given first time in existing literature.

The next is a modification of Example 9 from (Ampadu, 2017).

Example 7. Let $Y=[1,2]$ and $d_{Y}(u, v)=|u-v|^{2}$ and define $I$ : $Y \rightarrow Y$ by $I(u)=\frac{u+3}{4}$ for all $u \in Y$. Now observe that with $p=q=$ $\frac{1}{272}$ we have that (1) holds, i.e.,

$$
d_{Y}\left(I^{2}(u), I^{2}(v)\right) \leq \frac{1}{272} d_{Y}(I u, I v)+\frac{1}{272} d_{Y}(u, v),
$$

for all $u, v \in Y=[1,2]$.

Remark 8. In the past year, the new class of the $B$-metric space called Extended $B$-metric spaces is becoming actual for research. It is defined by applying

$$
\left(\mathrm{b}_{3}^{\prime}\right) \quad d_{Y}(u, w) \leq \Lambda(u, w)\left(d_{Y}(u, v)+d_{Y}(v, w)\right)
$$

instead of axiom $\left(\mathrm{b}_{3}\right)$ for every $u, v, w \in Y$, where $\Lambda$ is a function that maps $Y \times Y \rightarrow[0,+\infty)$. Obviously, every $B$-metric space is Extended $B$-metric.

Let us note that the previous considerations of $B$-convex contractions can be also treated in other classes of general metric spaces (partial metric spaces, $G$ metric spaces, $G_{B}$-metric spaces, $B$-metric spaces, extended $B$-metric spaces and many others...). 
In fact, even in the context of ordinary metric space, the following important questions have not been considered:

Question 1: What about the existence of the fixed point mapping in the case of complete metric spaces when the right hand side of the expression (1) is:
a) $k \max \left\{d_{Y}(u, v), \frac{1}{2}\left[d_{Y}(u, I u)+d_{Y}(v, I v)\right], \frac{1}{2}\left[d_{Y}(u, I v)\right.\right.$

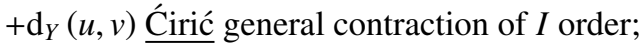

b) $k \max \left\{d_{Y}(u, v), d_{Y}(u, I u), d_{Y}(v, I v), \frac{1}{2}\left[d_{Y}(u, I v)\right.\right.$

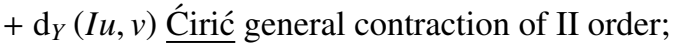

c) $k \max \left\{d_{Y}(u, v), d_{Y}(u, I u), d_{Y}(v, I v), d_{Y}(u, I v), d_{Y}(I u, v)\right\}$ Ćirić quasi-contraction.

Question 2: What about the existence of the fixed point mapping in the case of $B$-metric spaces when the right hand side of the expression (1) is:

a) $k \max \left\{d_{Y}(u, v), \frac{1}{2 B}\left[d_{Y}(u, I u)+d_{Y}(v, I v)\right], \frac{1}{2 B}\left[d_{Y}(u, I v)\right.\right.$ $+\mathrm{d}_{Y}(u, v)$

b) $k \max \left\{d_{Y}(u, v), d_{Y}(u, I u), d_{Y}(v, I v), \frac{1}{2 B}\left[d_{Y}(u, I v)\right.\right.$ $+\mathrm{d}_{Y}(I u, v)$

c) $k \max \left\{d_{Y}(u, v), d_{Y}(u, I u), d_{Y}(v, I v), d_{Y}(u, I v), d_{Y}(I u, v)\right\}$ Question 3: It is interesting to consider and the case if convex contraction $I$ is not continuous and if it is defined on a metric, that is, on a $B$-metric space.

Question 4: To consider the existence and uniqueness of the fixed point of continuous convex contraction of the third order, defined both on the ordinary metric and on the $B$-metric space. In this case the contraindicated condition has a form

$$
d_{Y}\left(I^{3} u, I^{3} v\right) \leq p d_{Y}\left(I^{2} u, I^{2} v\right)+q d_{Y}(I u, I v)+r d_{Y}(u, v),
$$

for all $u, v \in Y$, where $p, q, r \in[0,+\infty)$ and $p+q+r<1$ in the case of the metric space. Find a condition if $\left(Y, d_{Y}\right)$ is a $B$-metric space with a coefficient $B>1$. this field.

We conclude our work with another important question for

Question 5: Let $M$ and $N$ be a closed subsets in the complete metric space $\left(Y, d_{Y}\right)$ such that $I M$ is a subset of $N$ and $I N$ is a subset of $M$. If for all $u \in M$ and for all $v \in N$ it follows that

$$
d_{Y}\left(I^{2} u, I^{2} v\right) \leq p d_{Y}(I u, I v)+q d_{Y}(u, v),
$$

then the continuous mapping $I$ has a unique fixed point in crosssection of sets $M$ and $N$. Prove or disprove this claim by example.

Remark 9. Since the termcyclic type mapping was introduced in (Kirk et al., 2003) and thus generalized the famous Banach theorem, many researchers considered the newly introduced phenomenon and obtained corresponding results in various classes of general metric spaces. As far as we know from literature, this type of mapping has not been considered in the case of convex contractions of Istratescu. Therefore, the previous question is interesting for researchers working in this part of the non-linear analysis. Assuming that $M=N=Y$, a positive answer to the last question gives us as a consequence the main result of Istratescu. In other words, we would get a true generalization of Istratescu's result.

\section{ACKNOWLEGMENTS}

The Ministry of Education, Science and Technological Development of the Republic of Serbia supported the work of first author under contracts 174005 (Viscoelasticity of fractional type and optimization of shapes in rod theory) and 174024 (Methods of functional and harmonic analysis and PDE with singularities).

The second author is pleased to acknowledge the support of Distinguished Scientist Fellowship Program (DSFP), King Saud University, Riyadh, Saudi Arabia.

\section{REFERENCES}

Aleksić, S., Došenović, T., Mitrović, Z., \& Radenović, S. 2019a. Remarks on common fixed point results for generalized $\alpha_{*}-\psi$ contraction multivalued mappings in b-metric spaces, Adv. in Fixed Point Theory, 9 (1), pp. 1-16.

Aleksić, S., Kadelburg, Z. T., Mitrović, Z., \& Radenović, S. 2019 b. A new survey: Cone metric spaces. Journal of the International Mathematical Virtual Institute, 9, pp. 93-121.

Aleksić, S. T., Mitrović, Z., \& Radenović, S. 2019c. Picard sequences in b-metric spaces, too appear in Fixed Point Theory 2019-2020.

Alnafei, S. H., Radenović, S., \& Shahzad, N. 2011. Fixed point theorems for mappings with convex diminishing diameters on cone metric spaces. Applied Mathematics Letters, 24(12), pp. 2162-2166. doi:10.1016/j.aml.2011.06.019

Ampadu, C. K. 2017. On the analogue of the convex contraction mapping theorem for tri-cyclic convex contraction mappings of order 2 in b-metric space. J. Global Research Math. Archives, 4(6), pp. 1-5.

Ampadu, C. B. 2018. Some Fixed Point Theory Results For Convex Contraction Mapping Of Order 2. JP Journal of Fixed Point Theory and Applications, 12(2-3), pp. 81-130. doi:10.17654/fp0120230081

Andras, Sz. 2003. Fiber Picard operators and convex contractions. Fixed Point Theory, 4, pp. 121-129.

Bakhtin, I. A. 1989. The contraction mapping principle in quasimetric spaces. Funct. Anal, 30, pp. 26-37.

Collaço, P., \& Silva J. C. 1997. . A complete comparison of 25 contraction conditions. Nonlinear Analysis: Theory, Methods and Applications, 30(1), pp. 471-476. doi:10.1016/s0362546x(97)00353-2

Istratescu, V. I. 1981. Some fixed point theorems for convex contraction mappings and convex non-expansive mapping (I). Libertas Mathematica, 1, pp. 151-163.

Istratescu, V. I. 1982. Some fixed point theorems for convex contraction mappings and mappings with convex diminishing diameters. - I. Annali di Matematica Pura ed Applicata, 130(1), pp. 89-104. doi:10.1007/bf01761490

Istratescu, V. I. 1983. Some fixed point theorems for convex contraction mappings and mappings with convex diminishing diameters, II. Annali di Matematica Pura ed Applicata, 134(1), pp. 327-362. doi:10.1007/bf01773511 
Jeong, G. S., \& Rhoades, B. E. 2005.. Maps for which $\mathrm{F}(\mathrm{T})=\mathrm{F}\left(\mathrm{T}^{n}\right)$. FixedPointTheoryAppl, 6, pp.71 - 105.

irk, W. A., Srinivasan, P. S., \& Veeramani, P. 2003. Fixed points for R mappings satisfying cyclical contractive conditions. Fixed Point Theory, 4, pp. 79-89.

Kirk, W., \& Shahzad, N. 2014. Fixed Point Theory in Dis- tance Spaces.Cham: Springer Science and Business Media LLC. doi:10.1007/978-3-319-10927-5

Rhoades, B. E. 1977. A comparison of various definitions of contractive mappings. Transactions of the American Mathematical Society, 226, pp. 257-257. doi:10.1090/s0002-9947-1977-0433430-4 